\title{
O consumo de peixe anchoita na alimentação escolar: aceitabilidade e adesão
}

\author{
Consumption of anchovy fish in school meals: acceptability and adhesion
}

\author{
Simone Guerra Fonsecaa, Ana Luiza Sander Scarparob, Roberta Capalongac, Luciana Dias de Oliveirad, \\ Lauro Saint Pastous Madureirae, Vanuska Lima da Silva ${ }^{f}$ \\ a Nutricionista. Universidade Federal do Rio Grande do Sul (UFRGS). \\ b Nutricionista. Doutora em Educação e Ciências, UFRGS. \\ c Nutricionista. Mestre em Ciência e Tecnologia de alimentos. Centro Colaborador em Alimentação e Nutrição do Escolar, UFRGS. \\ d Nutricionista. Doutora em Saúde da Criança e do Adolescente. Docente do curso de Nutrição da UFRGS. Departamento de Nutrição. \\ e Graduação em Oceanografia Geológica e Oceanografia Biológica. Doutor em Hidroacústica aplicada a recursos marinhos. Fundação Universidade Federal do \\ Rio Grande (FURG). Departamento de Oceanografia, Laboratório de Tecnologia Pesqueira. \\ f Nutricionista. Doutora em Ciência dos Alimentos. Docente do curso de Nutrição da UFRGS. Departamento de Nutrição. \\ Fonte de Financiamento: Fundo Nacional de Desenvolvimento da Educação (FNDE).
}

Objetivo: Avaliar a possibilidade de inclusão do peixe Engraulis anchoita na alimentação escolar, considerando a aceitabilidade e a adesão, e os fatores que interferem em seu consumo pelos estudantes de escolas públicas no Rio Grande do Sul (RS).

Materiais e Métodos: Trata-se de um estudo transversal realizado em escolas públicas do Rio Grande do Sul, Brasil, que compreendeu duas etapas: a primeira analisando a aceitabilidade, pelo método Escala Hedônica, e adesão à preparação "massa com molho de anchoita"; a segunda avaliando a adesão em diferentes preparações com o peixe. Foi avaliada a frequência, absoluta e relativa, utilizando o Programa SPSS 18.0, onde se obteve os valores percentuais e médias de aceitabilidade e adesão dos itens em estudo. O projeto foi aprovado pelo Comitê de Ética em Pesquisa da Universidade Federal do Rio Grande do Sul sob n을 21550, atendendo a todos os critérios legais previstos para pesquisa científica com humanos.

Resultados: 1a etapa, aceitabilidade: participaram 26.533 escolares, sendo 10.169 de escolas estaduais, 6.278 de Rio Grande e 10.086 de Porto Alegre. 2a etapa, adesão: participaram 25.778 alunos de 77 escolas estaduais e 22 de Porto Alegre. Na etapa 1, verificou-se que 68, 71 e 81\% dos alunos, respectivamente, do Estado, Rio Grande e Porto Alegre gostaram da preparação. A adesão foi de $64 \%$ nas escolas estaduais e de $62 \%$ nas de Rio Grande, enquanto que nas de Porto Alegre foi de $45 \%$. Na etapa 2, a média de adesão foi de $92,1 \%$ para pizza, $72,3 \%$ para polenta, $87,5 \%$ para batata, $83,9 \%$ para pão, $72,9 \%$ para risoto e $68,5 \%$ para massa.

Conclusão: Acredita-se ser possível a inclusão do Engraulis anchoita na alimentação escolar por meio de iniciativas que promovam o consumo de pescado.

Palavras-chave: alimentação escolar; peixe; engraulis anchoita.

Objective: The aim of this study was to evaluate the possibility of including anchovy fish in school meals regarding acceptability and adhesion and the factors that lead to its consumption by students in public schools of Rio Grande do Sul.

Materials and Methods: The research was a cross-sectional study conducted in public schools of Rio Grande do Sul consisting of two stages: the first stage analyzed acceptability by means of the Hedonic Scale method and adhesion to "pasta with anchovy sauce"; the second stage evaluated student adhesion to different preparations with anchovy fish. Collected data was analyzed using SPSS 18.0 program, where the percentages of acceptability and adhesion were established. The project was approved by Universidade Federal do Rio Grande do Sul Ethic Committee by the number of 21550, taking into account all the legal criteria to scientific research on human beings.

Results: Step 1, acceptability: 26.533 schoolchildren participated in this phase, wherein 10.169 of whom were from state schools, 6.278 from Rio Grande, and 10.086 from Porto Alegre. Step 2, adhesion: 25.778 students from 77 state schools and 22 from Porto Alegre were part of this stage. In step 1, it was found that 68,71 and $81 \%$ of the students, respectively, from state schools, Rio Grande and Porto Alegre liked the preparation. Adhesion was of $64 \%$ in state schools and $62 \%$ in Rio Grande schools, while in Porto Alegre it was $45 \%$. In step 2, the average adhesion was $92.1 \%$ for pizza, $72.3 \%$ for polenta, $87.5 \%$ for potato, $83.9 \%$ for bread, $72.9 \%$ for risotto and $68.5 \%$ for pasta.

Conclusion: We believe it is possible to include anchovy fish in school meals through initiatives that promote fish consumption.

Keywords: school feeding; fish; engraulis anchoita. 


\section{INTRODUÇÃO}

O comportamento alimentar da criança começa a ser moldado no contexto familiar, por meio das suas experiências alimentares. $\mathrm{Na}$ idade escolar, o ato de se alimentar fica mais complexo, estando envolvidos fatores fisiológicos, psicológicos, sociais e culturais, além da fome, que passam a regular a ingestão de alimentos ${ }^{1}$. O ambiente de ensino, ao articular de forma dinâmica alunos e familiares, professores, funcionários e profissionais de saúde, proporciona as condições para desenvolver atividades que reforçam a capacidade da escola de se transformar em um local favorável à convivência saudável, ao desenvolvimento psicoafetivo e ao aprendizado, podendo, como consequência, constituir-se em um núcleo de promoção de saúde². Sendo assim, a escola é uma ferramenta fundamental para o desenvolvimento de ações que incentivem a formação de hábitos alimentares saudáveis, estimulando o consumo de frutas, hortaliças, cereais integrais e carnes magras, como o pescado ${ }^{3,4}$.

O pescado é um alimento que se destaca nutricionalmente quanto à qualidade e quantidade das suas proteínas, presença de vitaminas e minerais e, principalmente, por ser fonte de ácidos graxos essenciais ômega-3 eicosapentaenoico (EPA) e docosaexaenoico (DHA). Do terceiro trimestre até o segundo ano de vida, uma criança necessita de um suprimento constante de DHA para a formação do cérebro e de outras partes do sistema nervoso ${ }^{4,5}$. Apesar de seus benefícios comprovados à saúde, o Brasil apresenta um dos menores índices de consumo per capita de pescado do mundo. A média de consumo nacional é de 7 a $8 \mathrm{~kg} / \mathrm{hab} / \mathrm{ano}$, inferior ao recomendado pela Organização Mundial da Saúde, que é de $12 \mathrm{~kg} / \mathrm{hab} / \mathrm{ano}$, e à média mundial de 15,8 kg/hab/ano . Em relação à média de consumo do pescado na alimentação escolar, o per capita é de apenas 41,4 g/aluno/refeição7. Segundo Trondsen et al. ${ }^{8}$, o consumo de pescado em adultos e sua crença de que a alimentação deve ser saudável estão fortemente relacionados com a alta ingestão de peixe na infância. Entretanto, na pesquisa realizada em 2012 pelo Ministério da Pesca e Aquicultura (MPA), em parceria com o Fundo Nacional de Desenvolvimento da Educação (FNDE), sobre a inclusão do pescado na alimentação escolar, verificou-se que 638 (34\%) municípios afirmaram que houve inclusão do pescado na alimentação escolar, enquanto 1246 (66\%) alegaram não ter incluído peixe nos cardápios devido ao receio de acidentes com as espinhas e difícil preparo ${ }^{7}$, ou ainda por desconhecimento de outras formas de preparo. Por esse motivo, inúmeras iniciativas vêm sendo desenvolvidas a fim de estimular o consumo de peixe na alimentação escolar, aprimorando as condições de produção e processamento do pescado e aumentando a frequência da sua oferta 9 .
O peixe Engraulis anchoita pode ser considerado uma alternativa para a alimentação escolar, uma vez que apresenta altos teores de ácidos graxos poli-insaturados e proteínas com alta biodisponibilidade. A anchoita é um pescado de porte pequeno presente na região sul do Brasil, Argentina e Uruguai, com estimativas de população que variam entre 600.000 toneladas e 4.5 milhões de toneladas entre os três países ${ }^{10}$. Em função da morfologia da espécie, a rápida perda de frescor é a principal barreira tecnológica a ser transposta na sua inclusão em linhas industriais de processamento. Sendo assim, a elaboração de produtos a partir de base proteica de anchoita seria uma alternativa tecnologicamente viável para o aproveitamento em larga escala desse pescado ${ }^{11}$. A proposta de pesca e elaboração de produtos à base de anchoita para alimentação escolar conta com o apoio de ações governamentais, em nível nacional, por intermédio do MPA ${ }^{12}$. Além disso, a exploração da anchoita é realizada de forma sustentável, levando em consideração seu período de crescimento e reprodução ${ }^{10}$. A introdução da anchoita na alimentação escolar, portanto, poderá resultar em uma alternativa para aumentar o consumo de peixe pelos escolares, contribuindo assim para qualificação do cardápio e promoção de saúde na escola.

O presente estudo tem como objetivo avaliar a possibilidade de inclusão do peixe Engraulis anchoita na alimentação escolar, considerando a aceitabilidade e a adesão, e os fatores que interferem em seu consumo pelos estudantes de escolas públicas no Rio Grande do Sul (RS).

\section{MATERIAIS E MÉTODOS}

Esta pesquisa trata-se de um estudo transversal realizado em escolas públicas do Rio Grande do Sul, Brasil. A pesquisa foi desenvolvida no período de 2011 a 2014 e compreendeu duas etapas: a primeira analisando a aceitabilidade e adesão da preparação "massa com molho de anchoita" e a segunda avaliando a adesão ao peixe em diferentes preparações.

A anchoita enlatada utilizada foi produzida em decorrência da realização do projeto de pesquisa denominado "Pesca de Anchoita (Engraulis anchoita) com rede de meia água, na plataforma continental da região sudeste/sul do Brasil, processamento do pescado em terra e análise econômica da viabilidade da atividade ${ }^{\prime \prime 10}$, que desenvolveu, para uso exclusivo na alimentação escolar, a anchoita enlatada em molho com tomate, com peso líquido de $830 \mathrm{~g}$, sendo $500 \mathrm{~g}$ o peso drenado. Além disso, durante seu processo de industrialização, com o intuito de diminuir a quantidade de sódio no alimento final, foi retirada a etapa de salmouragem. As latas foram doadas pela Fundação Universidade Federal do Rio Grande (FURG) e a formalização da doação foi realizada diretamente com as secretarias de educação envolvidas - Secretaria de 
Educação do Estado do RS (SEDUC/RS), Secretaria Municipal de Educação de Porto Alegre (SMED/POA) e Secretaria Municipal de Educação de Rio Grande (SMED/RG).

Primeira Etapa: aceitabilidade e adesão da preparação "massa com molho de anchoita". O teste de aceitabilidade consiste no conjunto de procedimentos metodológicos, cientificamente reconhecidos, destinados a medir o índice de aceitabilidade da alimentação oferecida aos escolares ${ }^{13}$. Avaliou-se a aceitabilidade por meio do instrumento "escala hedônica", recomendado pelo $\mathrm{FNDE}^{14}$, que consiste no preenchimento pelos escolares de uma ficha que indica, em uma escala, o grau de satisfação ou insatisfação da preparação servida. O índice de aceitabilidade utilizando esse instrumento deve ser de, no mínimo, 85\%.

Foi realizado um teste piloto envolvendo duas escolas municipais, uma de Rio Grande e outra de Porto Alegre, a fim de verificar a metodologia e aprimorar a logística de coleta de dados. Em seguida, foram selecionadas as escolas da rede pública com mais de 100 alunos matriculados no ensino fundamental, sendo 60 escolas estaduais (de 44 municípios do Estado do RS), 35 municipais de Rio Grande e 46 municipais de Porto Alegre. Os testes de aceitabilidade e adesão ocorreram nas escolas estaduais do RS e municipais de Rio Grande, entre outubro e novembro de 2011, e nas escolas municipais de Porto Alegre, entre maio e julho de 2012. Foram utilizadas 7.750 latas do peixe anchoita em molho com tomate.

A partir da análise de cardápios do Estado e dos municípios, selecionou-se a "massa com molho de peixe" para padronização da preparação a ser ofertada, por ser usualmente fornecida e pela sua praticidade e viabilidade de oferta. A preparação foi adaptada para anchoita em molho com tomate e padronizada, por meio de ficha técnica de preparo. Os ingredientes foram adquiridos pelas escolas participantes, exceto as latas de anchoita, que foram fornecidas pela FURG. O preparo foi realizado nas próprias escolas pelos manipuladores de alimentos, que seguiram as instruções recomendadas na receita.

Segunda Etapa: adesão às diferentes preparações. O índice de adesão dos alunos à alimentação escolar corresponde à medida percentual de estudantes da escola que consumiram a alimentação preparada no dia do teste em relação ao total de alunos presentes ${ }^{13}$. A amostra consistiu-se de 218 escolas da rede pública de ensino do Estado do RS, sendo 155 da rede estadual e 63 da rede municipal de Porto Alegre. As escolas ofereceram a anchoita aos alunos no período de março a maio de 2014, e foram utilizadas 4.511 latas de anchoita em molho com tomate.

Levando-se em consideração os fatores que podem interferir na adesão dos alunos às preparações com anchoita, elaborou-se um formulário a ser preenchido pela escola e a receita com sugestões de preparações a serem servidas. No formulário, a escola foi questionada quanto ao município em que se localiza, rede de ensino (estadual ou municipal), preparação ofertada, número total de alunos matriculados, modalidades de ensino (infantil, fundamental, médio, educação de jovens e adultos - EJA), turnos das aulas, número de alunos presentes no dia da oferta do peixe e número de alunos que consumiram a preparação. As preparações sugeridas foram: pizza, massa, polenta, batata, pão e risoto com molho de anchoita. Cabe referir que as escolas também poderiam ofertar o peixe mais de uma vez e em diferentes preparações.

Segundo o manual para aplicação dos testes de aceitabilidade $^{13}$, os pontos de corte estabelecidos para determinar o índice de adesão são: Alto - acima de 70\%; Médio - 50 a $70 \%$; Baixo - 30 a 50\% e Muito Baixo - menor que $30 \%$. Foram preenchidos um total de 164 formulários, dos quais avaliou-se cada uma das variáveis que poderiam influenciar a adesão ao peixe, sendo: a rede de ensino, o tipo de preparação ofertada, a modalidade de ensino e os turnos em que foram servidas as refeições.

Foi avaliada a frequência, absoluta e relativa, por meio do Programa SPSS 18.0, onde se obteve os valores percentuais e médias de aceitabilidade e adesão dos itens em estudo.

O projeto foi aprovado pelo Comitê de Ética em Pesquisa da Universidade Federal do Rio Grande do Sul sob no 21550, atendendo a todos os critérios legais previstos para pesquisa científica com humanos.

\section{RESULTADOS}

Primeira Etapa: aceitabilidade e adesão da preparação "massa com molho de anchoita". Participaram desta etapa um total de 26.533 escolares matriculados no ensino fundamental, sendo 10.169 alunos de escolas estaduais do RS, 6.278 escolares do município de Rio Grande e 10.086 estudantes de Porto Alegre. Conforme apresentado na Tabela 1, verificou-se que uma parcela importante dos alunos respondeu que gostou ou gostou muito da preparação com anchoita oferecida pela escola. Já a adesão apresentou percentuais considerados médios nas escolas Estaduais do RS e municipais de Rio Grande, enquanto que nas escolas municipais de Porto Alegre a adesão foi baixa.

Tabela 1. Percentuais de aceitabilidade e adesão, de acordo com a rede de ensino, com relação à preparação "massa com molho de anchoita".

\begin{tabular}{lccc}
\hline \multicolumn{1}{c}{$\begin{array}{c}\text { Variáveis } \\
\text { em Estudo }\end{array}$} & Estadual/RS & Municipal/RG & Municipal/POA \\
\cline { 2 - 4 } Aceitabilidade & $68 \%$ & $71 \%$ & $81 \%$ \\
Adesão & $64 \%$ & $62 \%$ & $45,5 \%$ \\
\hline
\end{tabular}

RS: Rio Grande do Sul; RG: Rio Grande; POA: Porto Alegre. 
Tabela 2. Percentuais de adesão de acordo com a rede de ensino, estadual do RS ou municipal de Porto Alegre em relação às diferentes preparações ofertadas pelas escolas.

\begin{tabular}{lcccc}
\hline \multicolumn{1}{c}{ Preparação } & $\begin{array}{c}\text { No de ofertas } \\
\text { (RS-POA) }\end{array}$ & $\begin{array}{c}\text { Frequência geral por } \\
\text { preparação }\end{array}$ & $\begin{array}{c}\text { Frequência de adesão } \\
\text { escolas estaduais do RS }\end{array}$ & $\begin{array}{c}\text { Frequência de adesão } \\
\text { escolas municipais de POA }\end{array}$ \\
Pizza & $43(40$ RS-3 POA) & $92,1 \%$ & $88,4 \%$ & $95,8 \%$ \\
Massa & $73(56$ RS-17 POA) & $68,5 \%$ & $72 \%$ & $65,1 \%$ \\
Polenta & $18(18$ RS) & $72,3 \%$ & $72,3 \%$ & Não ofertado \\
Batata & $9(8$ RS-1 POA) & $87,5 \%$ & $75 \%$ & $100 \%$ \\
Pão & 6 (5 RS-1 POA) & $83,9 \%$ & $77,2 \%$ & $90,6 \%$ \\
Risoto & $11(11$ RS) & $72,9 \%$ & $72,9 \%$ & Não ofertado \\
Frequência geral & & & $76,3 \%$ & $87,8 \%$ \\
\hline
\end{tabular}

RS: Rio Grande do Sul; POA: Porto Alegre.

Segunda Etapa: adesão a diferentes preparações com molho de anchoita. Participaram 25.778 alunos de 99 escolas da rede pública de ensino, sendo 77 estaduais do RS e 22 municipais de Porto Alegre. As médias de adesão, de acordo com as modalidades de ensino, foram: $83,6 \%$ no ensino infantil; $72,9 \%$ no fundamental e $78,1 \%$ no EJA, sendo consideradas altas. Já no ensino médio foi encontrada adesão de $64,8 \%$, considerada média. Em relação à média de adesão por turno, foram obtidos os seguintes dados: $75,9 \%$ no turno da manhã; $72,6 \%$ no turno da tarde e $73,7 \%$ no turno da noite, considerada alta nos três turnos ${ }^{13}$.

A Tabela 2 apresenta os percentuais de adesão por preparação, por rede de ensino e o número de ofertas na rede estadual e municipal. Os percentuais considerados altos foram para pizza, polenta, batata com molho de anchoita, pão com molho de anchoita e risoto. No caso da massa, o percentual de adesão foi médio. Em relação às escolas da rede estadual e municipal de Porto Alegre, observa-se que em ambas a adesão foi elevada.

\section{DISCUSSÃO}

O pescado apresenta ácidos graxos poli-insaturados e proteínas de alto valor biológico, cuja digestibilidade pode chegar a ser maior do que das carnes em geral e do leite. Além disso, é uma boa fonte de vitaminas do complexo B, assim como A e D, no caso de peixes gordurosos. Quanto aos minerais, a carne de pescado é fonte de cálcio, fósforo, ferro, cobre e selênio, assim como possui um teor de sódio relativamente baixo ${ }^{5}$. Apesar desses benefícios, observa-se a pouca oferta de alimentos à base de pescado nas escolas do Rio Grande do Sul15. No entanto, alguns estudos ${ }^{6,16,17}$ demonstram que a introdução de produtos à base de peixe na alimentação escolar é uma alternativa viável, corroborando os resultados desta pesquisa.

A proposta de pesca e elaboração de produtos à base de anchoita para alimentação escolar se justifica por ser um alimento que apresenta alto valor proteico, baixo teor de sódio e rico em gordura ômega- $3^{10}$. O presente estudo demonstrou resultados promissores com relação ao pescado Engraulis anchoita na alimentação escolar. Embora abaixo do recomendado pelas metodologias de avaliação da aceitabilidade e adesão ${ }^{13}$, compreende-se que, de maneira geral, o peixe é um alimento não habitual na alimentação infantil, especialmente no Estado do RS, onde o consumo de carnes de origem bovina é de $66 \%$, enquanto que de pescado é de $2 \%$, como verificado em diferentes idades e classes sociais em três cidades da região sul, o que dificulta sua aceitação pelos alunos ${ }^{15,18}$. Francisco et al. ${ }^{19}$, caracterizando o perfil dos consumidores de frango no município de Porto Alegre, também observaram uma preferência por carne bovina de $60 \%$, sendo o frango a segunda opção de carne escolhida por esses consumidores, com $40 \%$ de preferência. Esses achados são confirmados pela Pesquisa de Orçamentos Familiares - POF, que ao analisar o perfil de consumo alimentar da população brasileira entre 2008 e 2009 observou um consumo per capita de 64,2g/dia de carnes de origem bovina, enquanto que o consumo per capita de pescado foi de $17,5 \mathrm{~g} / \mathrm{dia}^{20}$.

De acordo com os resultados obtidos, observa-se que a aceitabilidade da "massa com molho de anchoita", ofertada na primeira etapa, apresentou percentuais abaixo do recomendado para a metodologia utilizada, que é de $85 \%{ }^{13}$. As escolas da rede estadual e municipal apresentaram índices tanto de aceitabilidade como de adesão muito próximos, sendo que a adesão é considerada "média" (de 50 a 70\%).

Borges et al. ${ }^{4}$, estudando a aceitabilidade de nuggets assados e almôndegas cozidas do pescado Betara, obtiveram os índices de 92,4 e 89,4\% respectivamente, demonstrando que os dois produtos foram bem aceitos pelos escolares e, portanto, poderiam ser facilmente introduzidos na alimentação escolar. Em outro estudo, Valentim ${ }^{21}$ verificou a aceitabilidade das preparações "Peixe ao Brás" e "Risoto de Peixe" através dos métodos Escala Hedônica (EH) e Resto Ingestão (RI), obtendo uma média de aceitação de 87,4 e 
67,6\% na $\mathrm{EH}$ e 94,6 e 91,9\% no RI, respectivamente. Tais dados demonstram que o pescado pode ser utilizado em diferentes preparações e que, dentre essas encontram-se percentuais diferenciados de aceitabilidade e adesão, como apresentado neste trabalho.

As escolas municipais de Porto Alegre apresentaram o índice de aceitabilidade da massa com molho de anchoita mais próximo do preconizado (81\%). Esse fato pode ser atribuído à presença de pescado nos cardápios escolares do município. Segundo informações da SMED/POA ${ }^{22}$, são oferecidas preparações com peixe enlatado (sardinha) em média duas vezes ao mês, além de bolinho de peixe e filé de peixe, bimestralmente, sendo portanto, um alimento que faz parte da rotina dos estudantes, favorecendo sua aceitação.

Apesar da boa aceitação, neste caso, a adesão foi baixa, o que indica que apenas aqueles alunos que gostam de peixe consumiram a preparação. No entanto, outros fatores também podem estar relacionados à adesão a essa preparação, como a distribuição e horários em que as refeições são servidas, uma vez que as escolas municipais de Porto Alegre oferecem café da manhã, almoço e lanche da tarde. Diferentemente das escolas estaduais e municipais de Rio Grande, onde não há oferta de café da manhã e lanche da tarde e as refeições começam a ser servidas geralmente a partir das 10 horas, para o turno da manhã, e das 15 horas, para o turno da tarde. Embora esses não sejam os horários que usualmente a maioria da população consome uma refeição completa, muitos alunos aderem à alimentação escolar por ser a única opção de lanche oferecida na escola. Essa distribuição pode ter interferido na adesão à preparação ofertada devido ao curto intervalo entre as refeições, ou porque muitos alunos que chegam para o turno da tarde realizam a refeição do almoço em seus domicílios.

Quando avaliada a adesão às diferentes preparações com o peixe anchoita, na segunda etapa do estudo, pôde-se notar que a preparação "massa" foi a opção mais escolhida pelas escolas para ofertar aos alunos, devido, possivelmente, à sua praticidade de preparo, sendo oferecida 73 vezes nas diferentes escolas participantes, durante os três meses de estudo. Entretanto, a "massa" apresentou a menor adesão (68,5\%), considerada média ${ }^{13}$. A preparação que apresentou maior adesão foi a "pizza" (92,1\%), sendo a segunda opção mais escolhida pelas escolas para servir aos estudantes. A alta adesão a essa preparação pode ser explicada pelo fato de esse ser um alimento tradicionalmente incorporado nas preferências das crianças, o que vai ao encontro com o referido por Carvalho ${ }^{23}$, de que as escolhas alimentares não estão relacionadas apenas ao ato racional de comer, mas também aos seus significados.
Silva ${ }^{24}$ encontrou em Santa Catarina, Brasil, uma prevalência de $12,6 \%$ de consumo de pescado pelos escolares e ao estudar os fatores que influenciam esse consumo, identificou que os principais obstáculos foram o desgosto dos escolares pelo sabor do peixe, tipos de preparações e tipos de peixe ofertados nas escolas. Também foi referido que os alunos não possuem o costume de consumir esse alimento e que os professores não incentivam seu consumo. Sendo assim, observa-se a importância de ações de educação alimentar e nutricional que favoreçam as escolhas alimentares saudáveis dos escolares, conforme evidenciado por Fernandes et al. ${ }^{25}$ que observaram um impacto positivo dessas ações na redução do consumo de sucos artificiais e guloseimas e no aumento da ingestão de lanches nutritivos. Esses dados sugerem que seja possível o planejamento da alimentação como forma de promoção de práticas saudáveis, incluindo o consumo de pescado, o que pode exercer uma influência positiva nos fatores alimentares e comportamentais das crianças ${ }^{26}$.

Considerando-se o baixo consumo de pescado pela população brasileira, o alto valor nutricional que esse apresenta e os resultados obtidos nesta pesquisa, acredita-se ser possível a inclusão do pescado Engraulis anchoita na alimentação escolar por meio de iniciativas que promovam o consumo do mesmo. Ressalta-se a importância do estímulo à oferta de alimentos saudáveis no ambiente escolar, como frutas, hortaliças, cereais integrais e peixes, assim como ações de educação alimentar e nutricional nas quais os alunos tenham a oportunidade de entrar em contato com os alimentos e suas diferentes formas de preparo, a fim de reverter o quadro de monotonia alimentar dos cardápios escolares no que tange à variedade de formas de preparo e sabores de um mesmo alimento, possibilitando assim a construção e consolidação de práticas alimentares saudáveis.

Sugere-se que outros estudos a respeito de pescados no ambiente escolar sejam realizados, sobretudo em relação ao percentual de aceitabilidade, que é o mesmo para todos os tipos de alimentos e preparações e pode não representar a realidade em função da grande variedade de opções que esses podem ser ofertados.

\section{REFERÊNCIAS}

1. Brasil. Ministério da Saúde. Secretaria de Atenção à Saúde. Departamento de Atenção Básica. Dez passos para uma alimentação saudável: guia alimentar para crianças menores de dois anos: um guia para o profissional da saúde na atenção básica. 2ª ed. Brasília: Ministério da saúde; 2010.

2. Brasil. Ministério da Saúde e Ministério da Educação. Institui as diretrizes para promoção da alimentação saudável nas escolas de educação infantil, fundamental e nível médio das redes públicas e privadas, em âmbito nacional. Portaria Interministerial no 1.010, de 8 de maio de 2006. Brasília: Ministério da Saúde; 2006. 
3. Ramos FP, Silva SLA, Costa RAB. Educação alimentar e nutricional em escolares: uma revisão de literatura. Cad Saúde Pública. 2013;29(11):2147-61. https://doi.org/10.1590/0102$311 \times 00170112$

4. Borges NDS, Passos EDC, Stedefeldt E, De Rosso VV, Borges NS. Aceitabilidade e qualidade dos produtos de pescado desenvolvidos para a alimentacao escolar da Baixada Santista. Alim Nutr. 2011;22(3):441.

5. Sartori AGO, Amancio RD. Pescado: importância nutricional e consumo no Brasil. Segur Aliment Nutr. 2012;19(2):83-93.

6. Godoy L, Franco MLRS, Franco NP, Silva AF, Assis MF, Souza NE, Matsushita M, Visentainer JV. Análise sensorial de caldos e canjas elaborados com farinha de carcaças de peixe defumadas: aplicação na merenda escolar. Ciênc Tecnol Aliment. 2010;30(Supl.1):86-9. https://doi.org/10.1590/S0101-20612010000500014

7. Brasil. Ministério da Educação. Fundo Nacional de Desenvolvimento da Educação (FNDE). Dispõe sobre a inclusão de pescado na alimentação escolar. Nota técnica no 004. Brasília: Ministério da Educação; 2013.

8. Trondsen T, Braaten T, Lund E, Eggen AE. Consumption of seafood: the influence of overweight and health beliefs. Food Qual Prefer. 2004;15(4):361-74. https://doi.org/10.1016/S0950-3293(03) 00083-1

9. Brasil. Ministério da Pesca e Aquicultura. Dispõe sobre o incentivo à produção e comercialização de pescado [Internet]. [capturado 2015 Jan 15]. Disponível em: http://www.mpa.gov.br/index.php/ infraestrutura-e-fomento/144-incentivo-a-comercializacao

10. Pastous Madureira LS, Castello JP, Prentice-Hernández C, Queiroz MI, Espírito Santo ML, Ruiz WA, Raggi Abdallah P, Hansen J, Bertolotti MI, Manca E, Yeannes, MI, Avdalov N, Fernández Amorín S. Current and potential alternative food uses of the Argentine anchoita (Engraulis anchoita) in Argentina, Uruguay and Brazil. In: Hasan MR, Halwart M, editors. Fish as feed inputs for aquaculture: practices, sustainability and implications. Rome: FAO; 2009. p. 269-87. (FAO Fisheries and Aquaculture Technical Paper; 518).

11. Garcia-Torchelsen L, Jacob-Lopes E, Queiroz MI. Avaliação funcional de bases proteicas desidratadas de anchoita (Engraulis anchoita). Braz J Food Technol. 2011;14(4):283-93. https://doi. org/10.4260/BJFT2011140400034

12. Carvalho FM, Castello J. A pesca e o manejo da anchoíta: uma integração científica. In: Anais do V Simpósio Brasileiro de Oceanografia [Internet]. 2011 [capturado 2015 Mar 7]. Disponível em: http://www.vsbo.io.usp.br/trabs/137.pdf

13. CECANE-UNIFESP. Manual para aplicação dos testes de aceitabilidade no Programa Nacional de Alimentação Escolar PNAE: UNIFESP [Internet]. 2010 [capturado 2015 Jan 5]. Disponível em: http://www.fnde.gov.br/programas/alimentacao-escolar/ alimentacao-escolar-material-de-divulgacao/alimentacao-manuais/ item/5166-manual-para-aplica\%C3\%A7\%C3\%A3o-dos-testes-deaceitabilidade-no-pnae

14. Brasil. Ministério da Educação. Fundo Nacional de Desenvolvimento da Educação. Dispõe sobre o atendimento da alimentação escolar aos alunos da educação básica no âmbito do Programa Nacional de
Alimentação Escolar - PNAE. Resolução no 26 de 17 de junho de 2013. Brasília: Ministério da Educação; 2013.

15. Brasil. Ministério da Educação, Fundo Nacional de Desenvolvimento da Educação. Resumo Executivo. Composição Nutricional da Alimentação Escolar no Brasil: uma análise a partir de uma amostra de cardápios [Internet]. 2011 [capturado 2015 Abr 27]. Disponível em: http://www.fnde.gov.br/arquivos/category/116-alimentacaoescolar?download=8358: composicao-nutricional-da-alimentacaoescolar-do-brasil-pesquisa-de-cardapios-2011

16. Mitterer-Daltoé ML, Latorres JM, Carboneral N, Pastous-Madureira LS, Queiroz MI. Potencial de inserção de empanados de pescado na merenda escolar mediante determinantes individuais. Cienc Rural. 2012;42(11): 2092-98. https://doi.org/10.1590/S010384782012001100029

17. Donadini G, Fumi MD, SP. Hedonic Response to fish in preschoolers. J Sens Stud. 2013;(28):282-96. https://doi.org/10.1111/joss.12043

18. Silva FV, Prietch KM, Mendoça C, Chim JF. Características e Consumo de Pescado no Sul do Estado do Rio Grande do Sul. In: Anais do XXII Congresso de Iniciação Científica da Universidade Federal de Pelotas [Internet]. 2013 [capturado 2015 Fev 12]. Disponível em: http://cti.ufpel.edu.br/siepe/arquivos/2013/CA_01442.pdf

19. Francisco DC, Nascimento VP, Loguercio AP, Camargo L. Caracterização do consumidor de carne de frango da cidade de Porto Alegre. Cienc Rural. 2007;37(1):253-8. https://doi. org/10.1590/S0103-84782007000100041

20. Instituto Brasileiro de Geografia e Estatística. Pesquisa de orçamentos familiares 2008-2009: análise do consumo alimentar pessoal no Brasil [Internet]. 2011 [capturado 2015 Fev 13]. Disponível em: http://biblioteca.ibge.gov.br/visualizacao/livros/liv50063.pdf

21. Valentim DR. Testes de aceitabilidade de preparações com peixe em escolas públicas estaduais da Grande Vitória-ES [monografia]. Vitória: Faculdade Católica Salesiana do Espírito Santo; 2014.

22. Porto Alegre. Secretaria Municipal de Educação. Dispõe sobre os cardápios da alimentação escolar [Internet]. [capturado 2015 Maio 2]. Disponível em: http://websmed.portoalegre.rs.gov.br/escolas/ nutricao/

23. Carvalho MCVS, Luz MT, Prado SDP. Comer, alimentar e nutrir: categorias analíticas instrumentais no campo da pesquisa científica. Cienc Saúde Coletiva. 2011;16(1):155-63. https://doi.org/10.1590/ S1413-81232011000100019

24. Silva AM. Prevalência, fatores associados e barreiras ao consumo de peixes e frutos do mar em escolares do município de Florianópolis -SC [dissertação]. Florianópolis: UFSC; 2012.

25. Fernandes PS, Bernardo CO, Campos RMMB, Vasconcelos FAG. Avaliação do efeito da educação nutricional na prevalência de sobrepeso/obesidade e no consumo alimentar de escolares do ensino fundamental. J Pediatr (Rio J). 2009;85(4):315-21. http:// dx.doi.org/10.1590/S0021-75572009000400008

26. Cambraia RP, Murta NMG, Campos MHO, Botelho LA. Preferência alimentar de crianças e adolescentes: revelando a ausência de conhecimento sobre a alimentação saudável. Alim Nutr, Araraquara. 2012;23(3):483-9. 